\title{
El rol de las universidades y otras instituciones en el emprendimiento exitoso: visiones de una revisión de la literatura
}

\section{The role of universities and other institutions in successful entrepreneurship: Some insights from a literature review}

\author{
German A. Zarate-Hoyos ${ }^{1 \mathrm{a}}$ \& Fernando Larios-Meoño ${ }^{2 b^{*}}$ \\ ${ }^{1}$ State University of New York at Cortland, New York, Estados Unidos. \\ ${ }^{2}$ Universidad San Ignacio de Loyola, Lima, Perú. \\ ${ }^{a}$ Profesor asociado en SUNY-Cortland y profesor visitante en USIL. ${ }^{b}$ Jefe del Departamento de \\ Economía en USIL.
}

Recibido: $31-07-15$

Aprobado: $13-10-15$

*Correspondencia

Email: flarios@usil.edu.pe

Notas
Citar Como:

Zarate Hoyos, G., \& Larios Meoño, F. (2015). El rol de las universidades y otras instituciones en el emprendimiento exitoso: visiones de una revisión de la literatura. Propósitos y Representaciones, 3(2), 261-317. doi: http:// dx.doi.org/10.20511/pyr2015.v3n2.82

Este artículo se basa en un proyecto de investigación colaborativo que se llevó a cabo previamente entre la Universidad Estatal de Nueva York (SUNY) en Cortland y la Universidad San Ignacio de Loyola (USIL). Se presentó una versión previa de este trabajo en el Congreso Global en Administración de Empresas y Finanzas (GCBF) en San José, Costa Rica, del 26 al 29 de mayo del 2015. Los autores reconocen la asistencia de Thomas Lee, estudiante de Economía en SUNY-Cortland

(c) Universidad San Ignacio de Loyola, Vicerrectorado de Investigación y Desarrollo, 2015.

(cc) BY-NC-ND Este artículo se distribuye bajo licencia CC BY-NC-ND 4.0 Internacional (http://creativecommons.org/licenses/by-nc-nd/4.0/). 


\section{Resumen}

El objetivo de este artículo es analizar el rol de las universidades y otras instituciones en el emprendimiento exitoso. Se obtuvieron las diversas visiones sobre el tema a través de la revisión de la literatura. Estudios de casos de los Estados Unidos (emprendimientos Nueva York), España (Mondragón) y Alemania proveen pruebas claras de que las universidades son muy instrumentales en la creación, diseño e implementación de iniciativas empresariales al dar a los nuevos y actuales empresarios una formación en capital humano, fundamentos y modelos teóricos y empíricos para contribuir a los negocios que perduran en el tiempo. Los resultados de investigaciones sobre asuntos de género y riesgo muestran que las mujeres son menos propensas que los hombres a arriesgarse a trabajar e invertir en empresas nuevas. Otras instituciones, como los gobiernos, son decisivas en la facilitación de los incentivos necesarios para comenzar empresas nuevas, incluyendo recortes de impuestos, financiamiento de capital semilla, inversiones en capital humano, etc. Mientras la evidencia que apoya la relación entre la educación y los resultados empresariales es prometedora, aún no es definitiva. Además de ofrecer una revisión de investigaciones ya existentes, este artículo sugiere un marco teórico integrativo para investigaciones futuras.

Palabras clave: Emprendimiento, empresas nuevas, aversión al riesgo, correlación.

\section{Summary}

The purpose of this paper is to analyze the role of universities and other institutions in successful entrepreneurship. Insights are obtained following a literature review approach. Case studies from the United States (New York startup), Spain (Mondragon), and Germany provide strong evidence that universities are very instrumental in the creation, design and implementation of entrepreneurial initiatives by providing new and ongoing entrepreneurs with human capital training, fundamentals, and theoretical and empirical models to contribute to lasting businesses. Results from university research on gender and risk-issues lead to believe that women are more risk averse than men to work and invest in star-up businesses. Other institutions, such as governments, are critical to provide with the necessary incentives to launch 
start-up businesses, including tax cuts, seed capital funding, investment in human capital, etc. While the evidence supporting the links between education and entrepreneurial outcomes is promising it is not yet definitive. In addition to providing a review of existing research, this paper suggests an integrative framework for future research.

Keywords: Entrepreneurship, startup, risk-averse, correlation. 


\section{Introducción}

Durante los últimos años se ha dado mucha atención a los asuntos relacionados con los emprendimientos en el mundo empresarial desarrollado y en vías de desarrollo. A pesar del consenso general de cómo el espíritu empresarial contribuye a la economía a través de la generación de empleos, hay todavía muchas discusiones y dudas sobre su éxito duradero. Asimismo, la educación empresarial es una prioridad en las agendas políticas de los Estados Unidos y Europa, pero hay muy pocas investigaciones disponibles para evaluar sus efectos (Graevenitz, Harthoff \& Weber, 2010).

Parte de esta discusión puede centrarse en la manera en que se define el emprendimiento. Otro problema puede ser que el emprendimiento es visto como un tipo de solución que puede generar empleos e ingresos rápidamente, a veces llegando a arriesgar los fundamentos económicos que rigen los principios del mercado, así como la distribución eficiente de recursos para maximizar las ganancias a corto, mediano y largo plazo.

Existe evidencia de que la preferencia por la participación en nuevos emprendimientos se relaciona con la motivación personal para comenzar un nuevo negocio, la propensión a arriesgarse y la disponibilidad de otros ingresos. Esta evidencia también relaciona la productividad del tiempo de trabajo a dotaciones de capital humano, financiero y social y la prevalencia de actividades de subcontratación. Asimismo, la misma evidencia muestra que la mujer promedio invierte menos tiempo en el mundo de los negocios que los hombres, lo que se atribuye tanto a una menor preferencia por el tiempo de trabajo como a una menor productividad por hora trabajada (Verheul, Carree \& Thurik, 2008).

En este sentido, las universidades pueden tener un rol educacional clave para ayudar a facilitar la discusión sobre cómo se puede definir y entender el emprendimiento como un proceso. Las universidades también pueden ayudar a los emprendimientos (start-up) a construir modelos, poner en marcha sistemas y entrenar capital humano para que cubra las necesidades de su negocio de manera eficiente. 
Este artículo tiene definiciones, modelos, estudios de casos y lecciones que se aprendieron de experiencias en algunos países, y así contribuye a un mayor conocimiento del espíritu de emprendimiento.

\section{Definiciones de emprendimiento e iniciativas de emprendimiento.}

Un emprendimiento es una pequeña empresa de Starborn. Según Robehmed (2013), muchos fundadores de empresas tienen diferentes definiciones de lo que es un emprendimiento. Neil Blumenthal, codirector ejecutivo de Warby Parker, afirma que un emprendimiento es una compañía que trabaja para resolver un problema cuya solución no es obvia y cuyo éxito no está garantizado. El Street Journal reportó que, a nivel nacional, fracasan tres de cada cuatro emprendimientos, lo cual significa una tasa de éxito de apenas un $25 \%$. Se observa una tendencia similar en emprendimientos que provienen de programas incubadora.

Según Liddy (2010), del Syracuse Tech Garden, en Siracusa, Nueva York, la tasa de viabilidad a largo plazo para la incubadora de negocios Student Sandbox es de alrededor de un 39\%. Sin embargo, otros programas han tenido más éxito. Por ejemplo, el programa Acelerador de Negocios Techstars también ha tenido bastante éxito a nivel nacional, con un $75 \%$ de sus empresas aún activas.

Las incubadoras de negocios permiten que los emprendimientos nazcan y aumenten su probabilidad de éxito. Facilitan el apoyo a los negocios en varios aspectos, ofreciendo un lugar de trabajo y de orientación de mentores y expertos en el campo. También ayudan a conectar el futuro negocio con la asistencia financiera de inversionistas financieros. Respecto a la nación, la tasa de éxito de la Student Sandbox es mucho mayor. La tasa de éxito de Techstars es más impresionante y sobrepasa por mucho a la de la nación.

\section{La importancia de la educación en el emprendimiento.}

Un artículo de Dickson, Solomon y Weaver (2008) explora las relaciones entre la educación general, las formas específicas de educación empresarial y una gama de actividades empresariales en los Estados Unidos. Estas relaciones se investigaron a través de un análisis de investigaciones revisadas 
por otros expertos, que se publicaron en una gran variedad de periódicos y registros entre 1995 y 2006. Los resultados del artículo plantean una evidencia significativa que apoya la relación entre los niveles de educación general y varias medidas de éxito empresarial. Estos resultados son menos concluyentes en lo que respecta a la relación entre educación general y la elección de convertirse en empresario. Los resultados, que relacionan programas específicos de educación empresarial con el emprendimiento, a pesar de ser ambiguos, sugieren la existencia de una relación positiva entre dicha educación y tanto la elección de convertirse en empresario como el posterior éxito empresarial.

Un estudio reciente llevado a cabo por Arthur, Hisrich y Cabrera (2012) encontró que la educación calificaba muy arriba entre varios factores críticos investigados e identificados como facilitadores o impedimentos para un emprendimiento exitosamente global y regional. Este descubrimiento fue el resultado de una encuesta abierta y electrónica a 145 contactos de la industria. Las respuestas se categorizaron en tres grupos de factores, que son el económico, el social y el personal, y se analizaron de acuerdo con ellos por región y por las funciones de trabajo de quien respondía.

\section{Caso 1: El Modelo Estadounidense (el Caso de Nueva York).}

También ha habido un aumento notable en la educación empresarial en las dos últimas décadas. Los programas de educación y capacitación empresarial (ETT, por sus siglas en inglés) se ofrecieron en 400 universidades en 1995, comparado con más de 2,000 en los Estados Unidos en el 2012 (TomkinsBergh \& Miller, 2015).

Según la Fundación Nacional para la Ciencia, la colaboración industriauniversidad tiene muchas formas, y entre ellas: apoyo a la investigación, investigación cooperativa, transferencia de conocimiento y transferencia de tecnología (Chakrabarti \& Rice, 2003). También ha habido mecanismos efectivos desarrollados para asistir a los empresarios en las incubadoras de negocios a través de los años (Rice, 2002).

Se sabe que las universidades son los bastiones del aprendizaje y de la creación de conocimientos, y las colaboraciones datan de la Segunda Guerra 
Mundial, cuando se unieron a los esfuerzos para la guerra. Sin embargo, según Rice (2002), la tendencia en las universidades de los Estados Unidos ha sido hacia el desarrollo de conocimiento teórico y hacia un tipo de investigación más genérico. En particular, el autor señala que los fondos públicos rara vez pueden usarse para promover los intereses de una compañía específica. Por consiguiente, la alianza de compañías específicas que postulan a asociaciones colaborativas con universidades de los Estados Unidos bajo la iniciativa de Start Up NY no tiene los mecanismos estructurales para que les sea posible interactuar con los profesionales académicos en las universidades.

Otras iniciativas dignas de mención son los esfuerzos del programa Small Business Innovation Research (programa de Investigación para la Innovación en Pequeñas Empresas), en la Fundación Nacional para la Ciencia, para ayudar a las pequeñas empresas a promover sus actividades innovadoras, pero estos programas son escasos (Rice, 2002).

Según Chakrabarti y Rice (2003), algunas regiones de los Estados Unidos han intentado crear relaciones colaborativas eficientes entre universidades y corporaciones, pero han tenido éxito limitado en la mantención de dicho esfuerzo. Así, más iniciativas como Start Up NY son bienvenidas, basándose en el entendimiento de iniciativas exitosas alrededor del mundo y los roles tradicionales, pero que evolucionan, de las universidades y compañías hasta el momento.

El primero de enero del 2014, la oficina del gobernador Cuomo develó un programa llamado Start Up NY (estado de Nueva York, 2015), que crea y usa zonas libres de impuestos en los alrededores de las universidades neoyorquinas con la esperanza de atraer nuevos negocios y fomentar una economía creciente en Nueva York. El programa también contemplaba el uso de incubadoras para crear emprendimientos. La principal idea detrás de este plan es utilizar estas zonas libres de impuestos y con recortes de impuestos para fomentar que los negocios vengan a Nueva York y para impulsar el empleo. Traer negocios llevará a más inversiones en el estado y, ojalá, a un aumento significativo en el empleo. Otra parte del plan es promover las asociaciones entre negocios privados y universidades para fomentar negocios de alta tecnología y la innovación en el norte de Nueva York. Hoy por hoy se han creado alrededor de 395 puestos de trabajo, con unos US\$50 millones de inversión en el estado. 
Mientras que Start Up NY incluye incubadoras de negocios, el programa también ofrece otros múltiples beneficios. Los nuevos negocios que comienzan en Nueva York tienen ciertos beneficios tributarios desde su creación o reubicación. Los beneficios incluyen la exoneración de impuestos a la renta, a la propiedad y a las empresas por 10 años. Asimismo, sus nuevos empleados (se esperan hasta 10,000 nuevos puestos de trabajo por año) no pagarán impuestos por al menos cinco años. Esto se extiende a 10 años si los trabajadores ganan menos de US\$200,000. La idea es aumentar la rentabilidad de los negocios para que puedan aumentar los empleos. También se espera que haya efectos multiplicadores, pues los nuevos empleados tienen más dinero en los bolsillos debido a los impuestos más bajos, dinero que gastarán en la economía local y regional, incrementando la actividad económica y el empleo. Sin embargo, estos negocios sí tienen algunas restricciones antes de recibir los beneficios.

Las restricciones y requerimientos para que una empresa en Nueva York califique para Start Up NY son relativamente simples. Primero, el negocio debe ser una compañía que cree nuevos puestos de trabajo en Nueva York. No se permite al nuevo negocio trasladar trabajadores de otros lados. Esto asegura que haya un incremento neto del empleo en Nueva York. Debido a este requerimiento, los negocios preexistentes en Nueva York no pueden postular. Asimismo, si el negocio está en la Ciudad de Nueva York, Long Island o Westchester County, el negocio debe ser un emprendimiento en alta tecnología. Si un negocio quisiera estar cerca de la ciudad, la fabricación no es una opción. Más aún, muchos tipos de negocios no son una opción, incluyendo la venta al detalle, alimentos, empresas médicas o dentales, bufetes de abogados, contadores, producción de energía, finanzas, etc. En segundo lugar, hay un requerimiento de terreno/zonificación. La universidad debe ser propietaria del terreno, y el negocio tiene que ubicarse, como máximo, a una milla del campus universitario. Las universidades escogen las empresas, que deben estar alineadas con la misión de la universidad, y ellas mismas tienen un límite. Las universidades no pueden reubicar programas existentes, estudiantes, profesores o empleados para dejar el espacio para un nuevo negocio. 


\section{Caso 2: El Modelo Europeo (el Modelo Mondragón).}

El sistema educacional en Mondragón está menos separado del mundo laboral. Hay bastante interacción entre trabajadores, gerentes y administrativos académicos en el Consejo Rector, el cual está conformado por miembros de cada grupo. Esta participación va en ambas direcciones; esto significa que los miembros de la universidad participan en consejos de rectores de negocios y viceversa. Estas interacciones crean una relación mucho más cercana y receptiva cuando se trata de prácticas, asignaciones, financiamiento, currículum, etc. Esto también se traduce en algunas prácticas que se observan, como la alternación de trabajo y estudios para más de 3,500 estudiantes en los últimos seis años, los proyectos de fin de curso en varias compañías en el sistema para 350 estudiantes al año, aproximadamente, y la experiencia tradicional de trabajo y prácticas de enseñanza para más de 500 estudiantes al año.

La Universidad de Mondragón parece haber combinado los valores de un sistema educacional de humanidades con el mundo laboral al incorporar no solo el conocimiento técnico específico requerido para los diplomas y grados, sino también los aspectos relacionados con las competencias genéricas y los valores humanos en todo el modelo educacional. También buscan participar en la economía global a través del marco de una educación multilingüe y el uso de tecnologías de la comunicación y de la información.

En la declaración de objetivos en la página web de la Universidad de Mondragón se lee "nuestro modelo de enseñanza incluye un sistema de relaciones que, con el sistema educacional como el tema principal, apunta hacia la inclusión de las compañías e instituciones en el área, para así garantizar la accesibilidad social, la combinación de trabajo y estudios, el desarrollo de la investigación y la provisión de Educación Continua”. Por lo tanto, no es sorprendente que el desarrollo de la investigación sea uno de los pilares del sistema educacional en la Universidad de Mondragón. Esto forma la base para la creación de una red sólida de instituciones de apoyo como Ikerlan e IDEKO, que son dos centros tecnológicos reconocidos internacionalmente, y la promoción del Polo de Innovación Garaia para fomentar el desarrollo conjunto de la investigación. En este contexto, el desarrollo de empresas nuevas parece ser un resultado lógico más que una directiva que viene desde 
arriba. Los valores del emprendimiento emanan de la búsqueda constante de nuevas ideas y de nuevos acuerdos institucionales de diferentes participantes para combinar trabajo y estudios y permitir a los estudiantes ponerse en contacto directo con el mundo laboral desde el comienzo de sus estudios.

Existe otro modelo para promover emprendimientos no en los Estados Unidos, sino en España. La Corporación Cooperativa Mondragón (CCM) en el País Vasco español es uno de los grupos empresariales españoles líderes. La CCM se compone de cooperativas autónomas e independientes con 257 subsidiarias y cooperativas de producción, 74,060 personas, 15 centros de tecnología y oficinas corporativas en 41 países. Cinco estudiantes de un sacerdote llamado José María Arizmendiarrieta comenzaron este grupo en los años 50. Arizmendiarrieta enseñó a un grupo de estudiantes en una escuela politécnica, que no podía entrar al capitalismo local, decidiendo formar su propia cooperativa. En ese momento no había ningún precedente legal para lo que habían hecho y no eran elegibles para recibir dinero del estado para la creación de su nuevo negocio. Los cinco miembros originales facilitaron el dinero inicial y los recursos financieros hasta 1975, donaciones que recolectaban en el camino y, más tarde, contratos con clientes. Fundaron ULGOR, la primera cooperativa de Mondragón. Desde entonces, Mondragón ha sido el ejemplo modelo de las cooperativas que quieren sobrevivir en el mundo capitalista. Se ha dicho que Mondragón no sacrifica la equidad por la eficiencia y que tampoco sacrifica la eficiencia por la equidad (Flecha \& Santa Cruz, 2011). El grupo Mondragón tiene dos instituciones principales que tratan con la creación de nuevos negocios (Saiolan e Ikerlan), y su experiencia fue informativa para Start Up NY.

Saiolan es la parte de la Corporación Cooperativa Mondragón orientada a la creación de empresas y fundada en 1981 como parte de la Escuela Politécnica de Ingeniería. Saiolan actúa como la incubadora de negocios y brinda asistencia para que los nuevos negocios florezcan. Saiolan asiste en el lanzamiento de nuevas empresas, cooperativas o cualquier otro modelo de negocios, y en la diversificación de negocios ya existentes. También trabaja en el desarrollo regional, estudios de viabilidad, así como en estudios tecnológicos y de patentes. Forma empresarios en cinco áreas: motivación, encuentro de oportunidades, definición de proyectos adecuados, planificación de nuevos negocios y lanzamiento de la idea. Saiolan enseña 
a los empresarios cómo lanzar su proyecto, permitiendo a la gente trabajar en equipos, trabajar abiertamente, y proporcionando asistencia y asesoría. Es interesante mencionar que Saiolan no es una cooperativa per se, pues eso haría que no pudiese recibir fondos del gobierno vasco. En lugar de ello, se mantiene como una corporación y puede recibir subsidios y préstamos del gobierno vasco para financiar empresas nuevas. De hecho, una buena parte del dinero no viene sólo del gobierno vasco, sino que son sus contribuciones junto con las de Mondragón y del Consejo Provincial en Gipúzkoa que, combinadas, equivalen al 30\% del presupuesto de Saiolan. El otro $70 \%$ viene de proyectos en curso con negocios locales/regionales, así como de la Unión Europea. Este programa educacional informal no tiene exámenes formales, y su meta es el lanzamiento de un negocio exitoso y la creación de líderes de empresas con capacidades empresariales. Los participantes también pueden tener una idea de negocios sin ser necesariamente estudiantes (Ifateyo, 2011). Los investigadores de Saiolan evalúan las ideas, las encuentran viables o inviables, y luego comienza la investigación. Los emprendedores consiguen un espacio para trabajar, financiación de Saiolan y un cuerpo técnico.

Ikerlan cabe en la categoría de investigación/desarrollo de productos. Ikerlan está ligada a Mondragón por ser parte del grupo CCM y fue creada en 1974 por un grupo de empresas cooperativas. Es un centro de desarrollo e investigación tecnológica que se enorgullece de estar a la vanguardia de la investigación en varias áreas, como eficiencia energética, eficiencia operacional, desarrollo de productos y servicios, y tiene como objetivo tener tecnología de punta. También ofrece la promoción/producción de una variedad de productos. Colaboran con clientes de diversos campos, comenzando con una idea, y luego el proceso se dirige al diseño de producto y, después, hacia el desarrollo de producto. Científicos y técnicos profesionales llevan a cabo las operaciones técnicas, así como la fase previa al diseño, y no los estudiantes, que son supervisados por estos profesionales. La fase previa al diseño se centra en desarrollar los productos de manera correcta.

Con estos elementos siendo parte del proceso, el producto y las estrategias tecnológicas llevan al desarrollo tecnológico y del producto, más lo que Ikerlan llama OIC (oportunidades, ideas y conceptos u “Oh, I see!") o actividades previas al desarrollo. Se agrega más a este proceso compartiendo conocimientos (una idea popular en Mondragón) y compartiendo estrategias, 
así como el conocimiento tecnológico y de productos, y conocimientos de gestión en varios niveles. El control de gestión está para no formalizar de más la actividad de desarrollo. La prefase ha sido bien afinada para satisfacer las necesidades de Ikerlan, sus socios y sus clientes (MacGregor, Araba, Parra \& Lorenzo, 2006). Esto es ahora menos un programa educacional que un programa de desarrollo. El financiamiento viene predominantemente de contratos y, algo, del gobierno vasco, el Ministerio de Educación, Ciencia y Tecnología, gobiernos municipales y la Unión Europea (Shantz \& Macdonald, 2013). Ya que Ikerlan es una organización sin fines de lucro, todas las ganancias excedentes se reinvierten para el desarrollo y la investigación. Esto les permite mantenerse un paso adelante por sobre la competencia e invertir en tecnología de vanguardia.

Mientras que ambas instituciones participan en el desarrollo de potenciales ideas comercializables, tienen aproximaciones más bien distintas en estos dos procesos diferentes. Ikerlan se centra más en usar su energía para crear o promover un éxito. Saiolan se involucra en el trabajo en equipo con el objetivo de crear algo nuevo usando varias mentes. Estas dos instituciones son increíblemente exitosas, teniendo Ikerlan 500 millones de euros (684 millones de dólares) en valores acumulados de sus emprendimientos. En el 2013, el presupuesto de Saiolan fue de un millón de euros. Ambas organizaciones trabajan con grandes corporaciones y, de manera importante, con la Unión Europea en varios emprendimientos. Saiolan ha tenido su gran cuota de éxito, especialmente si se le mira en términos de negocios exitosos creados y que continuaron después de que Saiolan terminara con sus contribuciones. De las 103 empresas creadas por Saiolan, 100 han prosperado y sobrevivido por más de 30 años. Solo eso es una tasa de éxito impresionante que supera el $97 \%$. Esto fue posible incluso durante los tumultuosos años de opresión de Franco sobre el pueblo vasco. Más recientemente, entre 1985 y 2013, se crearon más de 200 empresas y 2,500 nuevos puestos de trabajo gracias a Saiolan. La implicancia es que los negocios que comienzan bajo estos acuerdos institucionales funcionan de manera continua porque la gente gastará más su dinero en la economía local y regional, y ese dinero inducirá una mayor actividad económica (aún a un nivel pequeño) bajo una onda de efectos multiplicadores a través de la economía. 


\section{Explicación del éxito del grupo Mondragón.}

Hay varias razones por las que Mondragón, Saiolan e Ikerlan han tenido la oportunidad de conseguir éxito. Sin embargo, creemos que las razones se pueden resumir en tres ideas principales.

Coordinación institucional. Una razón por la que a Mondragón le ha ido tan bien es la creación de una red, más que de una sociedad, con varios sectores diferentes, como el financiero, el académico y el de negocios, y la utilización de esta red a su máximo potencial. Bajo un autogobierno, el grupo Mondragón es muy democrático y las decisiones se toman en equipo, en vez de hacerlo una sola persona o unas pocas personas. Tienen estatutos sobre su negocio y la manera en que debería funcionar. Han sido muy creativos resolviendo problemas, llegando a crear su propio banco con el único propósito de ayudar a empresas cooperativas y continuar encontrando otros acuerdos financieros para ayudarse más a ellos mismos. Trabajan de cerca con el gobierno local y el resto de sectores en su red. Esto tiene como resultado una mayor eficiencia y productividad. Estar tan interconectados también ayuda a aquellos que pueden perder su trabajo en una parte de la cooperativa, reubicándolos en otra parte o, posiblemente, incluso en una parte externa de la red. Esto mantiene alto el empleo en el área, racionalizando varios procesos y manteniendo bajos los costos globales.

Financiación. Como indica Henderson-Harr (2015), el financiamiento de muchos de estos programas de Nueva York en general pasará por las universidades. Una diferencia principal entre la experiencia del sistema SU NY y de Mondragón (Saiolan e Ikerlan) es cómo se asigna el dinero. Ikerlan y Saiolan reciben un financiamiento adecuado, según lo consideran los participantes de su incubadora de negocios y el centro de investigación y desarrollo sin fines de lucro . Las incubadoras en Nueva York reciben financiamiento del estado y tienen algo de ayuda de Start Up NY (según John de SS), pero tienen una financiación mucho menor que Saiolan. Estos centros de investigación y desarrollo deberían identificar áreas claves y solicitar financiamiento suficiente para invertir en tecnología de vanguardia. Las incubadoras también deberían estar provistas del financiamiento adecuado y del espacio para que las nuevas empresas puedan operar. El gobierno vasco, los gobiernos locales, y hasta los cuerpos más grandes como la 
Unión Europea, financian a Ikerlan. Con una mayor financiación, continúan teniendo acceso a tecnología de vanguardia. Esta tecnología resulta en más innovación, productos y servicios de mayor calidad, que permiten al grupo competir de manera más efectiva tanto regional como globalmente.

\section{Por último, el éxito de Mondragón puede atribuirse en gran medida}

a la cultura del pueblo vasco. Esto se debe tanto a motivos económicos como no económicos. Respecto a los términos no económicos, los vascos son un grupo muy orgulloso. Tienen mucho orgullo étnico. Se consideran vascos y no españoles, y han intentado más de una vez ser completamente autónomos respecto a España. Según Making Mondragon, por Whyte y Whyte (1991), tienen mucha dignidad en su trabajo. Después de más de 60 años en funcionamiento, su logo aún dice "Humanity at Work", humanidad en el trabajo. Ponen el 100\% en todo lo que hacen, incluso en el trabajo más pequeño. Asimismo, combaten la alienación del trabajo haciendo que cada trabajador sea dueño y rotando a sus trabajadores dentro y fuera de trabajos menores, entre otras cosas. Sin embargo, esto viene de la historia, pues han participado en el mundo de los negocios por varios siglos. En el siglo XIV, los vascos participaban activamente en la construcción de barcos. Cuatro siglos después, participaban activamente en la producción de acero. El día de hoy compiten a nivel global como la Corporación Cooperativa Mondragón.

Económicamente hablando, ser importante en el mundo de los negocios por siglos crea conocimiento sobre contratos, la gestión de flujos financieros hacia dentro y hacia afuera. Aunque la confianza es una razón no económica, al final la gente pone dinero en las manos de las compañías en que confía. Muchos pueden confiar en Mondragón debido a su trayectoria y a que los vascos están fuertemente involucrados en la producción de acero. Más aún, el día de hoy, su legislación da subsidios y dinero a Mondragón, así que la falta de inversión no parece ser un problema. Aunque la cultura en su totalidad es una razón compleja para su éxito general, también es, sin duda, un factor importante en la producción de negocios y emprendimientos. A las visiones e ideas individualistas en lugares como los Estados Unidos a menudo se les reconoce con un ambiente en el cual los emprendimientos pueden tener éxito y las personas pueden seguir lo que se conoce como el Sueño Americano. A las personas con ideas en lugares como los Estados Unidos a menudo se les reconoce con algo nuevo y toman riesgos y tienen 
altas probabilidades de éxito. En varios lugares, como España y Canadá, las cooperativas proporcionan números con viabilidad a largo plazo más alta que los negocios individuales.

El individualismo puede ser la causa base de la motivación para emprender un negocio, pero el éxito también parece venir de los valores desinteresados de un medio cooperativo, así como de la presencia de una red fuerte (leyes cooperativas). Estos valores han apoyado las ideas innovadoras en el lugar de trabajo, tales como el plan de contingencia para el alejamiento de los trabajadores, y también han ganado la confianza y el apoyo del sector político, lo cual ha asegurado su éxito. Según una entrevista de Democracy NOW (2013) con Mikel Lezamiz, director de Diseminación Cooperativa en Mondragón, la cultura/valores de Mondragón son inculcados en las mentes del pueblo vasco desde temprana edad. Aunque la universidad puede no tener directamente un curso denominado Diseminación Cooperativa, dice Lezamiz, trataría de enseñar a los alumnos y educarlos en valores cooperativos, valores que son también transpersonales al pueblo vasco desde temprana edad. Sin embargo, ellos también son miembros de las cooperativas y, por lo tanto, el grupo puede tener un grupo potencial de trabajadores futuros con valores cooperativos.

\section{Economía de la educación, género y problemas de riesgo en el emprendimiento: dos estudios de caso alemanes.}

Un primer estudio de caso alemán escrito por Verheul, Carree y Thurik (2008) analizó los efectos separados de preferencia y productividad en el número de horas de trabajo en nuevos emprendimientos de empresarios hombres y mujeres, siguiendo un enfoque cuantitativo y usando un modelo de dos ecuaciones no lineales.

Una primera relación del modelo tuvo como variable dependiente el número de horas invertidas en la compañía en 1994. Una segunda relación del modelo consideraba las ganancias como la variable dependiente. Las variables independientes del modelo eran: otros ingresos, género, edad, propio jefe, aversión al riesgo, educación, vocación, experiencia, trabajo similar, capital de emprendimiento, existencia de contactos con otros empresarios. 
Los resultados de los autores mostraron que "las personas tienen una menor preferencia por el tiempo de trabajo si tienen otros ingresos disponibles, y los empleados tienen mayor aversión al riesgo, y no están motivados para emprender un negocio siendo sus propios jefes. La productividad del tiempo está definitivamente relacionada con el capital financiero invertido, la industria y la experiencia relevante, el contacto con otros empresarios, el número de empleados, el manejo de una empresa existente, el tener instalaciones comerciales separadas y el predominio de actividades de externalización".

Los autores descubrieron que, en promedio, las mujeres invertirían menos horas en la compañía que los hombres debido a una menor preferencia promedio por el tiempo de trabajo y a una menor productividad por hora trabajada. Ellos creen que la menor preferencia de las mujeres por el tiempo de trabajo se puede explicar por la disponibilidad de otros ingresos y la naturaleza de aversión al riesgo de las mujeres, mientras que la menor productividad femenina se asocia a niveles menores de capital humano, social y financiero. En su análisis de las ganancias, los autores encontraron un efecto negativo del género en las horas de trabajo y en las ganancias. Más aún, ellos creen que las expectativas de los empresarios con respecto a los factores que influyen en su productividad no coinciden completamente con su efecto real en las ganancias.

Un segundo estudio de caso alemán escrito por Caliendo, Fossen y Kritikos (2010) analiza cómo las actitudes de riesgo pueden influir en el ciclo de vida completo de los empresarios. Los autores proponen que, mientras investigaciones recientes respaldan la proposición teórica de una correlación positiva entre las actitudes de riesgo y la decisión de ser autoempleado, los efectos sobre la supervivencia no son tan directos. Desde una perspectiva psicológica, el estudio supone una relación inversa en $U$ entre las actitudes de riesgo y la supervivencia empresarial. Utilizando datos validados experimentalmente del Panel Socioeconómico Alemán (SOEP), los autores examinan la medida en la cual las actitudes de riesgo influyen en las tasas de autoempleo en Alemania. Los resultados confirman que las personas cuyas actitudes de riesgo se encuentran en un rango medio sobreviven como empresarios por mucho más tiempo que las personas con actitudes de riesgo particularmente bajas o altas. 


\section{Lecciones de Mondragón y los casos alemanes para la iniciativa de emprendimiento de Nueva York.}

Al observar el éxito de Mondragón se pueden obtener varias lecciones para ayudar al éxito de Start Up NY. Para que las incubadoras en Nueva York incrementen su tasa de éxito a números cercanos a los de Saiolan, se debe hacer una mayor inversión. Aunque el presupuesto para la Student Sandbox no es público, su programa tiene apenas 12 semanas. En España, el programa de Saiolan dura hasta dos años. Este periodo, asignado a aquellos involucrados, permite la creación de empresas y el tiempo para que crezcan y maduren. Les da tiempo y recursos para establecerse y evitar la alta tasa de fracaso de las nuevas empresas y para aumentar la probabilidad de éxito a largo plazo. Saiolan tiene un mejor financiamiento y, por lo tanto, puede permitirse invertir en un periodo de tiempo más prolongado para ver el desarrollo del negocio. Más aún, también pueden ayudar a empresas preexistentes a diversificarse, mientras que la mayor parte de las incubadoras en Nueva York sólo se enfocan en la creación de emprendimientos. En la página web de Student Sandbox, la diversificación de los negocios no forma parte de sus objetivos. No obstante, la intervención en esta área también puede conducir a una mayor tasa de éxito, ya que las empresas se crearán para durar en el tiempo.

Mientras que existe la necesidad de un mejor financiamiento de las incubadoras de negocios, si Nueva York planea incorporar negocios de alta tecnología, también necesitamos incrementar el financiamiento de nuestros centros de investigación y desarrollo. Existen cuatro centros principales en el sistema SUNY. Estos centros están en Albany, Binghamton, Rochester, y Buffalo. Ikerlan es un centro de investigación de vanguardia que se autosostiene y no tiene fines de lucro. Reinvierte todo el dinero que gana en tecnología de punta y obtiene la mayor parte de sus ingresos de contratos comerciales con el resto que viene de la Unión Europea y el Estado y del gobierno vasco y de gobiernos municipales. Nuestros centros de investigación en Nueva York son financiados exclusivamente por el Estado. Además, según Fauci (2014), los fondos de US\$900,000 de Nueva York son, en gran medida, menores que el dinero del presupuesto de Ikerlan. 
El presupuesto de Ikerlan es de alrededor de US\$22,000,000 (reporte anual de Ikerlan). Una idea para financiar este centro sería tomar el dinero necesario para el recorte tributario a fin de crear más centros que se alineen con las fortalezas de la región o disminuir algunos de los recortes tributarios masivos ofrecidos e invertir en los cuatro centros principales existentes para fomentar la innovación. La inversión en un proyecto como este permite el uso de nueva tecnología e innovación para empresas nuevas y preexistentes para ayudarlas a ser más competitivas. El punto clave es que el financiamiento del gobierno es necesario y vital para el éxito de este programa, ya que no llegará del sector privado debido a externalidades positivas. En la literatura económica, este fenómeno se conoce como falla de mercado.

También puede ser necesaria una gestión financiera más robusta. Mondragón creó la Caja Laboral, que era un banco utilizado para ayudar a fundar empresas cooperativas. Por ejemplo, Brasil tenía un programa similar llamado Directrizes da Politica Industrial, en el cual el gobierno proporcionaba dinero y recortes tributarios a las empresas, lo que ayudaba al éxito del programa. El uso de zonas libres de impuestos es solo el principio. Se debe crear un acuerdo financiero más sólido a fin de que Start Up NY sea más útil en la creación de emprendimientos.

Como organización sin fines de lucro, Ikerlan tiene un objetivo: mantenerse como una institución de investigación de vanguardia. ¿Cómo se logra esto? Las utilidades generadas se reinvierten. El dinero no sale de la compañía, sino que se reinvierte en Ikerlan para mantenerla en la cima. Sobre todo, en Mondragón, una gran parte del dinero queda dentro. Los fondos solo parecen salir cuando un trabajador se jubila o fallece. Como escribieron Flecha y Santa Cruz (2011), gran parte del dinero se queda dentro. Esto, al igual que para Ikerlan, permite a Mondragón innovar y crear nuevas ideas como su propio banco (Caja Laboral), un parque científico como el Syracuse Tech Garden y la Universidad de Mondragón.

Fuera de las sociedades de la empresa y universidad, la creación de sociedades con las incubadoras de negocios locales podría ser beneficiosa para los programas académicos. Los emprendimientos podrían utilizar los recursos de la escuela, y esta aun ganaría por el ingreso de las matrículas. También, cuando estos dos sectores trabajan juntos, se comparten los 
costos de la tecnología. Además, Saiolan y la Universidad de Mondragón trabajan juntos, con los empleados de Saiolan laborando allí el 70\% del tiempo y un $30 \%$ en la Universidad de Mondragón. Esto se podría utilizar de manera similar aquí, pero quizás en un orden inverso. Permitir que algunos profesores universitarios apoyen a los estudiantes/empresarios potenciales en sus emprendimientos podría ser altamente beneficioso, pero requiere de una estructura flexible en ambas partes. La idea principal, tanto de esta sociedad como de la expansión de las clases de emprendimiento y el uso de prácticas, es crear sinergias entre los participantes para desarrollar algo más grande de lo que pueden hacer individualmente. Cuando se establece una relación estrecha entre el mercado laboral y las universidades, estas también pueden recibir una valiosa retroalimentación para mejorar sus programas . Permitir la cooperación entre empresas y universidades con la selección de un historial práctico/de campo en el currículum para algunas especialidades puede incrementar el valor educacional del grado. La probabilidad de encontrar un empleo adecuado una vez terminados los estudios también puede incrementarse.

Se necesita más que solo conectar las empresas con las universidades. El grupo Mondragón creó una red en la cual, según Uribe (2014), un trabajador de Saiolan utiliza una red que cubre universidades, empresas, instituciones financieras, gobierno e instituciones de investigación, entre otros. Esto reporta diversos beneficios. En primer lugar, reduce los costos e incrementa la eficiencia. Cada uno de estos vínculos ofrece diversos beneficios para cooperar estrechamente entre sí. Desde luego, no todo es una historia de éxito, como lo ha demostrado recientemente el fracaso de FAGOR, la cooperativa original, que entró en quiebra. La pérdida de cualquier negocio tiene costos sociales, pero se lidia con estos de manera interna a través de diversos mecanismos. La compañía de seguros interna para Mondragón, Lagun Aro, le permite a Mondragón proporcionar hasta el $80 \%$ del reemplazo de salarios por beneficios de desempleo, lo que es más que el $60 \%$ que da el gobierno federal de los Estados Unidos. Además, la organización de la cooperativa brinda más oportunidades después de tener una mala experiencia como esta. Los trabajadores tienen más opciones, como el desempleo por ciclos (donde algunos empleados son desvinculados de la empresa por un año en lugar de serlo permanentemente), la capacidad de iniciar una nueva cooperativa o, simplemente, cambiarse a otra cooperativa o empresa. Los despidos son 
inusuales gracias a la elaborada red que han creado, la cual proporciona seguro de desempleo, desempleo por ciclos, etc., y la oportunidad de encontrar un nuevo empleo apropiado. Estos planes de contingencia menores por sí mismos aminoran los efectos financieros y económicos que acarrea el desempleo. Muchas personas, como Fred Freundlich, de la Universidad de Mondragón, atribuyen a la red antes mencionada el potencial de vínculos institucionales creados por Mondragón. No hay motivo para que el éxito de Ikerlan no se logre en otras partes.

Fuera del mundo empresarial está el mundo académico, el cual puede contribuir al éxito de Start Up NY. En primer lugar, la expansión de las clases de emprendimiento en las universidades del norte de Nueva York sería un buen punto de partida. Por ejemplo, durante una reciente visita, uno de los autores observó que la Universidad de Mondragón tiene un programa de postgrado que permite a los estudiantes crear un emprendimiento con la ayuda/recursos de la universidad sin clases en el currículum y pidiendo, como único requisito para graduarse, un cierto número de ventas de un producto nuevo. Este ambiente permite a los estudiantes sentirse en la total libertad de ser creativos, de tener mentores a medida y de interactuar con otros para intercambiar y experimentar con ideas. Parte del financiamiento de Start Up NY fue asignado para crear clases de emprendimiento en SUNY Cortland y en otras instituciones de educación superior en el sistema SUNY. En Cortland, en particular, las clases eran electivas más que requisitos pero, en el futuro, pueden ser parte de un grupo o subespecialidad. Estas clases son de campo y permiten a los estudiantes trabajar en grupos y desarrollar planes de negocios similares al programa de la Universidad de Mondragón. Hasta el momento, los estudiantes de la primera promoción en el centro de Cortland crearon la tienda de Bagel Long Island.

En segundo lugar, el incrementar las prácticas ofrecidas por empresas locales también podría ser beneficioso al trabajar en una red. Las universidades en Nueva York también pueden hacer que las prácticas sean un requisito, como lo son en otros estados. Esto permitirá a las empresas contar con una cantidad regular de practicantes. El modelo también puede incluir especializaciones que no son de negocios bajo una opción electiva. La revista Forbes señala que el $37 \%$ de los practicantes no remunerados y el $60 \%$ de los practicantes remunerados obtuvieron una oferta de trabajo después de 
graduarse (Adams, 2012). Esto ocurre comúnmente en Mondragón, donde profesores y gerentes de cooperativas trabajan juntos como estudiantes en una de sus numerosas prácticas requeridas. Los estudiantes deben hacer hasta el 30\% de su trabajo en clase en las cooperativas (Freundlich, 2015). Mientras que esto puede parecer excesivo, muy bien puede crear una fantástica relación entre el mundo de los negocios y la academia. También debemos agregar la competencia global a la capacitación a través de diversas modalidades de estudio en el extranjero o prácticas internacionales. Los estudiantes de idiomas pueden conectarse globalmente, pero, en general, también otras especialidades pueden tener oportunidades de participar en el aprendizaje global, como la iniciativa SUNY COIL, para traer el aprendizaje de red global a las universidades de los Estados Unidos.

En tercer lugar, otra lección aprendida de Saiolan e Ikerlan es abrirse al público en general. Mientras que Saiolan utiliza estudiantes, también está abierto al público en general. Algunas instituciones dan créditos por experiencias de vida. Si este es el caso, expándase esa idea y hágase que todas las universidades de Nueva York acepten experiencias de vida para obtener créditos. Si no es por los créditos, se debe permitir que las personas que no son estudiantes o los alumnos que no estudian negocios desarrollen ideas y, potencialmente, emprendan negocios nuevos localizados. Los estudiantes de negocios no tienen el monopolio de las ideas de emprendimiento. Al expandir la red a otros miembros de la comunidad, se puede utilizar un reservorio de ideas mucho más rico para el desarrollo de nuevos negocios.

La Universidad de Mondragón y Saiolan tienen una particular diferencia que los distingue de la iniciativa de Nueva York: ubicación. La iniciativa restringe los negocios al área que está en/dentro de una milla del campus universitario, que es de propiedad de la universidad. En lugar de imponer un límite, estas dos optan por abrirse. Además, el programa de Brasil a escala nacional tampoco tiene restricciones en cuanto a la ubicación de los programas. Invirtió en el programa y en las sociedades entre centros de investigación y desarrollo. En Pensilvania también se llevó a cabo un programa similar, que tenía un límite más amplio, de un radio de hasta 4 millas (Henderson-Harr, 2015). El requisito de ubicación constituye una de las mayores críticas al programa de Nueva York, y muchos, incluyendo al presidente Bitterbaum, de SUNY Cortland, la 
comparten. Otro tema controversial son las restricciones impuestas por el estado con respecto al tipo de negocio.

Finalmente, siempre existe controversia con respecto a los impuestos. El gravar con ciertos impuestos a las empresas nuevas podría aliviar algo de presión al estado. Hay muchos estados que tienen códigos y tasas tributarias más efectivas que Nueva York: 49 de ellos. Según Tax Foundation (n.d.), Nueva York es el quincuagésimo estado con peores impuestos en la nación. Esto plantea un problema: lo que les pasa a los negocios que están operando actualmente y que no obtienen las nuevas tasas impositivas. Por ejemplo, muchos empresarios locales en Cortland, que tienen años de experiencia en el campo, creen que muchas empresas deben salir del estado y moverse hacia el sur, compensando cualquier nueva creación de empresas a través de este programa. Sin el ingreso por impuestos y la pérdida de ingresos por los recortes tributarios, el estado tiene el potencial de terminar en un peor estado financiero. Crear un ambiente tributario más amigable de modo generalizado puede ser un mejor plan. En Brasil, el gobierno, por lo general, ofrece recortes tributarios, aunque a veces los elimina todos. Por otro lado, Mondragón entra a un mercado con impuestos altos. Sin embargo, luchan por crear un campo de juegos justo en vez de ofrecer una ventaja competitiva a un grupo selecto de empresas que tienen la suerte de estar en el área.

Saiolan y la Universidad de Mondragón también se diferencian en términos de sus expectativas de los negocios creados en oposición a la iniciativa de Nueva York. Saiolan trabaja con un grupo cooperativo para crear empresas. Estas empresas tienden a no tener restricciones en lo que producen. Las empresas que ayudan a crear pueden tener fines de lucro o no; pueden ser una corporación, una cooperativa o cualquier otro tipo de organización. Además, según señala Uribe (2014), han ayudado a crear empresas que van desde sistemas de acceso a pinchos/tapas (pequeños platos de comida) en la región vasca para ser vendidos en San Sebastián. Además, han ayudado a muchas otras empresas, como de dispositivos antirrobos para autos, aplicaciones para teléfonos móviles y estabilidad urbana. Sin embargo, las regulaciones pueden restringir la efectividad del programa de Nueva York. Según The Economist (2014), las empresas están más dispuestas a pagar impuestos si no existen sobrerregulaciones en estos programas. Start Up NY ofrece áreas libres de impuestos a las 
nuevas empresas, pero impone muchas restricciones y una gran cantidad de "sobrerregulaciones".

Actualmente, no hay ninguna parte que actúe como vínculo entre las universidades y la cámara de comercio. Sin embargo, esta necesidad se satisface gracias a reuniones regionales en cada una de las 10 regiones del estado de Nueva York. El propósito de dichas reuniones es facilitar las comunicaciones entre las instituciones y las agencias de desarrollo económico en cada región. Estas reuniones también permiten el compartir ideas y el presentar tratos potenciales dentro del programa Start Up NY. Además, una pregunta que queríamos responder era si existía la cooperación entre las universidades a través del programa SUNY para compartir activos como la tecnología y programas para desarrollarlo y para progresar más. Según el contacto, hoy no existe tal programa a través de la misma iniciativa, pero esto no significa que no se formen relaciones externas actuales con este propósito en una base programa-a-programa. Muchas relaciones se forman dentro de un contexto similar. Creo que estos dos puntos podrían estar impidiendo el progreso del programa. Aunque las reuniones brindan tiempo fundamental para las instituciones y las agencias de desarrollo económico, la utilización de un vínculo que trabaje directamente entre las universidades/empresas y la cámara de comercio podría ayudar mucho al programa y agilizar el proceso financiero. También, implementar un mecanismo que ayude a compartir la tecnología y los programas dentro de la iniciativa podría agilizar el proceso de compartir dichas tecnologías y aminorar el potencial desperdicio de dinero y tiempo valioso para todas las partes implicadas. Con un proceso así se llegaría a los acuerdos de manera previa, y el proceso completo sería más fácil. Las cooperativas de la Experiencia Mondragón trabajan de manera similar y a menudo colaboran en varios proyectos distintos. Estos proyectos pueden ser para Mondragón, la Unión Europea o la sociedad en general (Freundlich, 2015).

Saiolan usa diversas métricas para medir su éxito, como el número de empresas o empleos creados. Pero, tal vez, la mejor métrica disponible que se puede usar para medir el éxito sería la tasa de viabilidad a largo plazo. Según el Departamento del Trabajo (2014), en los Estados Unidos, en un periodo de cinco años, solo el $50 \%$ de las empresas sobrevive sin ninguna ayuda. Con la asistencia de la incubadora Student Sandbox, los 
emprendimientos que de ella surgen tienen una tasa de viabilidad a largo plazo del 39\%. Esto es una mejora, pero con más financiación y más ayuda de la creación de una red, y con financiamiento para la investigación y el desarrollo, esta tasa puede crecer exponencialmente hasta alcanzar números como los que Mondragón ha obtenido. Mondragón es una mejora, pero con un mayor financiamiento y una mayor ayuda para la creación, la tasa ha sido del $80 \%$. Se logró este porcentaje en medio de una recesión económica en Europa. Su éxito se debe a los acuerdos financieros de las compañías, así como a varias de sus políticas, que ya hemos descrito. Si Nueva York utiliza esto como métrica, se puede ver qué tan efectiva ha sido su inversión en lo que respecta a los negocios viables creados, así como a los nuevos empleos creados. Más emprendimientos crearán más trabajos, y con más trabajos habrá más actividad económica a través de sus efectos multiplicadores. Start Up NY tiene el potencial para ser un programa exitoso; sin embargo, tiene que tratar ciertos problemas planteados por las partes involucradas.

Finalmente, los casos alemanes sugieren que los programas de emprendimiento deben prestar atención a los problemas de género. Los resultados que indican que las mujeres invierten menos horas en una empresa cuando existe otro ingreso, junto con el resultado de que las mujeres tienen una mayor aversión al riesgo, señalan que se deben crear incentivos adicionales para que ellas se unan a las filas de los nuevos empresarios. Sin embargo, los resultados que muestran que solo las actitudes de tomar riesgos medianos incrementan significativamente las oportunidades de supervivencia de un negocio demuestran que las mujeres pueden tener aversión al riesgo, pero que no necesitan tomar grandes riesgos para dedicarse a las actividades empresariales. No obstante, puede existir la necesidad de crear distintos programas de mentores y redes adicionales a fin de asegurar el éxito de las mujeres en las actividades empresariales. Estos resultados también se deben tomar en cuenta en los cursos de emprendimiento, ya que las estudiantes mujeres algunas veces superan el número de estudiantes varones en la educación superior, de manera que se espera ver una mayor cantidad de féminas en tales cursos. 


\section{Conclusiones y sugerencias de políticas}

\section{Conclusiones.}

Mondragón y sus instituciones, como Saiolan e Ikerlan, constituyen un gran modelo en lo que respecta a los ingredientes clave en los programas exitosos para fomentar la creación/emprendimientos de negocios. En particular, los vínculos institucionales entre distintos participantes son fundamentales para la creación exitosa de nuevos negocios que perduren. Tal como se señalara antes, los dos centros de innovación, Saiolan e Ikerlan, proporcionan un apoyo indispensable para la experiencia exitosa que es Mondragón. Ellos permiten que el grupo Mondragón sea competitivo en España y a nivel global. Mondragón es único en cuanto a la creación de sus vínculos institucionales y se ha beneficiado, en gran medida, de su existencia.

Nueva York se ha embarcado en una gran empresa con Start Up NY, pero el programa se restringe a ciertos negocios, y la ubicación geográfica muy limitada de las empresas puede crear un sistema dual entre las compañías que se benefician de los recortes tributarios y aquellas que no lo hacen. Asimismo, hace falta financiamiento para las incubadoras y actividades de apoyo relacionadas, pues este es escaso. Finalmente, las relaciones institucionales entre los participantes son pocas.

El caso alemán sugiere que las mujeres enfrentan retos distintos a los hombres en el lugar de trabajo, los cuales determinan su participación en actividades empresariales. En particular, los bajos niveles de capital humano, social y financiero pueden restringir su participación en nuevas empresas. Por lo tanto, los problemas de género se deben considerar en el currículum de los cursos de emprendimiento. Estos mismos problemas se deben tratar en los programas que patrocinan las actividades de emprendimiento.

\section{Sugerencias de políticas.}

El aprender del experimento de Mondragón, así como de otros experimentos en el país y en el extranjero, puede determinar los factores clave para hacer que Nueva York sea un mejor lugar económico y financiero en general. 
La vinculación de líderes empresariales con funcionarios universitarios de manera más regular, y con relaciones institucionales sólidas, puede contribuir al éxito de Start Up NY a largo plazo. Más allá de la creación de nuevas empresas, el resultado se puede extender al diseño de una educación fuertemente orientada al emprendimiento en los centros universitarios. Se debería apoyar un marco institucional para la interacción continua de los líderes empresariales y los funcionarios universitarios.

Una mayor inversión en nuestros centros de investigación preexistentes reduce los costos de su creación y distribuye el dinero de manera más eficiente, lo cual puede ser increíblemente efectivo, tal como se ve en Brasil. El financiamiento adecuado de las incubadoras de negocios, competencias de emprendimiento y otras actividades de apoyo también puede contribuir a la creación de una cultura del emprendimiento más allá de las aulas.

También se debe tener en cuenta que el aumento del empleo y el fomento de una mejor economía no son tan simples como utilizar zonas libres de impuestos y confiar en la reubicación de negocios cerca de las universidades. El gobierno (tanto federal como estatal) debe invertir fuertemente y debe haber menos regulaciones pero más efectivas. Las reglas injustas que se crean por tener algunas empresas sin pagar impuestos, mientras que las empresas existentes siguen teniendo algunas de las tributaciones más altas del país, deben tratarse quizás manteniendo a los negocios a través del ofrecimiento de reducciones de impuestos (pero no exoneraciones completas) y proporcionando asistencia técnica a la universidad, lo cual se menciona más adelante con mayor detalle.

Ya que muchas de estas actividades vinculan a las universidades con empresas existentes o potenciales, corresponderá a las universidades crear programas de emprendimiento, ya sea como especialidad dentro de un programa de negocios o como una posible subespecialidad para estudiantes que no son de negocios. Aunque comenzar y manejar una práctica requiere de principios básicos de emprendimiento, desde la investigación de mercado, pasando por la ubicación clave, hasta la viabilidad financiera, estos deben considerarse. Las universidades también pueden efectuar más investigaciones con respecto a los factores que afectan la toma de riesgos o la aversión al riesgo entre la población, ya que este es un aspecto 
fundamental que determina el hecho de que una persona se involucre o no en un emprendimiento. Financiar la investigación que incluye los problemas de género es también muy necesario, porque los resultados hasta el momento no parecen ser concluyentes. Se pueden identificar los patrones de consumo con la ayuda de la psicología para tratar el comportamiento del consumidor.

Finalmente, los departamentos universitarios que participan en la tecnología de vanguardia deben buscar directamente sociedades con empresas privadas o, bien, crear vínculos institucionales que establezcan una red similar a Mondragón para promover empresas rentables cuyas utilidades se puedan reinvertir en empresas similares.

\section{Referencias}

Adams, S. (2012, July 25). Odds Are Your Internship Will Get You a Job. Forbes. Retrieved from: http://www.forbes.com/sites/susanadams/2012/07/25/ odds-are-your-internship-will-get-you-a-job/

Arthur, S. J., Hisrich, R. D. \& Cabrera, A. (2012). The importance of education in the entrepreneurial process: a world view. Journal of Small Business and Enterprise Development, 19(3), 500-514.

Fauci, D. (2014). State budget lays foundation for new pharmacy school. Binghamton University. Retrieved from http://www.binghamton.edu/ inside/index.php/inside/story/8277/state-budget-lays-foundation-fornew-pharmacy-school/

Caliendo, M., Fossen, F. \& Kritikos, A. (2010). The impact of risk attitudes on entrepreneurial survival. Journal of Economic Behavior and Organization, 76(1), 45-63.

Chakrabarti, A. \& Rice, M. (2003). Changing Roles of Universities in Developing Entrepreneurial Regions: The Case of Finland and the US: MIT, IPC.

Democracy Now (2013). Interview with Mickel Lezamiz on the 25th March 2013. Understanding the Mondragon Worker Cooperative Corporation in Spain. [Video file]. Retrieved from http://www.democracynow. org/blog/2013/3/25/video_understanding_the_mondragon_worker cooperative_corporation_in_spains_basque_country 
Department of Labor (2014, May 7). Entrepreneurship and the U.S. Economy. Retrieved from http://www.bls.gov/bdm/entrepreneurship/bdm_chart3. htm.

Dickson, P. H., Solomon, G. T. \& Weaver, K. M. (2008). Entrepreneurial selection and success: does education matter? Journal of Small Business and Enterprise Development, 15(2). 239-258.

Flecha, R. \& Santa Cruz, I. (2011). Cooperation for Economics Success: The Mondragon Case, Analyse \& Kritik, 33(1), 157-70. Retrieved from http://www.analyse-und-kritik.net/en/2011-1/AK_Flecha_SantaCruz 2011.pdf

Freundlich, F. (2015). Interview of Fred Freundlich via telephone by Thomas Lee.

Henderson-Harr, A. (2015). Personal interview with Amy Henderson-Harr at her SUNY Cortland office on February 2015.

Graevenitz, G., Harhoff,D.\& Weber, R.(2010). The effects of entrepreneurship education. Journal of Economic Behavior and Organization, 76(1), 90112 .

Ifateyo, A. N. (2011, May 18). Mondragon Training Journal: Saiolan, Mondragon's Business Innovation Center. Mondragon Training Journal: Grassroots Economic Organizing.

Liddy, John (2010, March 29). Syracuse Technology Garden entrepreneur in residence provides support for student ventures. SU News.

MacGregor, S. P., Araba, J., Parra, I. \& Lorenzo, Ma. P. (2006). Supporting New Product Creation in the Mondragon Valley. European Journal of Innovation Management, 9(4), 418-443.

New York State (2015, January 15). 2015 Opportunity Agenda. Talk delivered by A. Cuomo in Albany, NY.

Rice, M. P. (2002). Co-production of business assistance in business incubators: An exploratory study. Journal of Business Venturing, 17, 163-187.

Robehmed, N. (2013, December 16). What is a StartUp? Forbes. Retrieved from http://www.forbes.com/sites/natalierobehmed/2013/12/16/what- 
is-a-startup/

Shantz, J. \& Macdonald, J. B. (2013). Beyond Capitalism. USA: Bloomsbury Academic.

Tax Foundation (n.d.). New York, The Facts on New York's Tax Climate. Retrieved from http://taxfoundation.org/state-tax-climate/new-york

The Economist (2014, July 5). Red Tape Blues. Small Businesses fret less about taxes than over-regulation. Retrieved from http://www.economist. com/news/united-states/21606293-small-businesses-fret-less-abouttaxes-over-regulation-red-tape-blues.

Tomkins-Bergh, C. \& Miller, A. (2015). Entrepreneurship Education and Training: What Works. Kauffman Foundation. Retrieved from http:// www.kauffman.org/blogs/growthology/2015/07/entrepreneurshipeducation-and-training

Uribe, I. (2014). Interview with Isabel Uribe by German Zarate during the May 2014 Workshop on the Mondragon Cooperatives in Mondragon, Spain.

Verheul, I., Carree, M. \& Thurik, R. (2008). Allocation and productivity of time in new ventures of female and male entrepreneurs. Discussion Papers on Entrepreneurship, Growth.

Whyte, W. F. \& Whyte, K. K. (1991). Making Mondragon: The Growth and Dynamics of the Worker Cooperative Complex, ILR Press, 2sd edition. September, 1991. 